\title{
Frontiers in plant senescence research: from bench to bank
}

\author{
Su-Sheng Gan • Stefan Hörtensteiner
}

Published online: 12 July 2013

(C) Springer Science+Business Media Dordrecht 2013

Senescence in plants refers to unique terminal active biological processes leading to the ultimate attrition of a leaf, a flower, a fruit or even a whole plant. In our view, as shown below, senescence has two "domains": one is the "sense" domain and the other is the "science" domain, especially when an "I" is inserted (to "inhibit" senescence), it forms a perfect "science" domain.

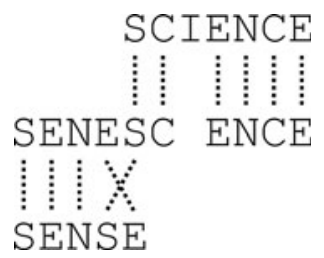

Recent research on senescence in plants has focused on these two domains, that are (1) to make SENSE of senescence at the molecular levels, and based on the molecular findings, (2) to devise ways to inhibit or manipulate senescence for agricultural improvement; in other words, to translate laboratory bench findings to practical applications. And much progress has been made in the past several years. The purpose of this special issue is to review and update the most recent research developments in these frontiers.

The visible sign of senescence in leaves and in some fruits is the breakdown of chlorophyll. The underlying biochemical and molecular mechanisms have been very actively investigated and significant progress has been

\section{S.-S. Gan $(\bowtie)$}

Departments of Horticulture, and Plant Breeding and Genetics, Cornell University, Ithaca, NY 14853, USA

e-mail: SG288@cornell.edu

\section{S. Hörtensteiner}

Institute of Plant Biology, University of Zürich,

CH-8008 Zürich, Switzerland made. Hörtensteiner (2012) has now offered an update in this regard. Systems biology is a new holistic approach to many aspects of biomedical and biological processes including plant senescence, and Guo (2012) has summarized the most current understandings of leaf senescence resulting from the use of this approach. Epigenetics represents another most hot area in life science. Humbeck (2012) reviews the current evidence that leaf senescence is regulated through epigenetic processes. Thus, several epigenetic mutants were shown to exhibit a senescence phenotype and the processes to be involved include global chromatin structure changes, senescence-specific histone modifications and changes in DNA methylation. It is well known that senescence can be induced and regulated by an array of environmental cues (such as drought stress, darkness, and extreme temperatures) and endogenous factors (such as reproductive growth, plant growth regulators, and age). How these signals are perceived and transduced has remained as hot topics. Zhang and Zhou (2012) and Jibran et al. (2013) have brought us the recent advances in signal transduction and hormonal regulation integrated with developmental and stress signals. It should be noted that age-related changes or ARCs, has been used by gerontologists and other scientists for many years. In their review, Jibran et al. (2013) have defined "an ARC as any irreversible change that is strictly dependent on age and thus development" and based on several lines of evidence, have proposed that hormonally and environmentally induced leaf senescence processes depend on ARCs. This is an interesting yet debatable hypothesis.

In addition to leaf senescence, significant progress has recently also been made in senescence of flowers and fruits. In her review on flower senescence, Rogers (2012) updates research in understanding environmental, developmental and hormonal signals that affect petal senescence. In 
addition, signal transduction pathways such as MAP kinase cascades and downstream cellular processes such as proteolytic and autophagic processes begin to be better understood. Gapper et al. (2013) review most recent research advances in fruit ripening and senescence, with a focus on the underlying molecular and hormonal (especially ethylene) regulatory mechanisms. After harvest, fruits continue their senescence processes, and recently much effort has been made to unravel the regulatory processes and mechanisms. In their review, Tian et al. (2013) brought us the most current biochemical and molecular understandings of senescence and related pathogenesis in harvested fruits, with an emphasis on reactive oxygen species or ROS.

In addition to basic research, we have also witnessed considerable progress in translational research on plant senescence. There are many lines of evidence that plant senescence is highly related to crop yield. The review of Gregersen et al. (2013) discusses different attempts that have been undertaken to manipulate senescence. They list many examples of mutants and crop cultivars that show an association between leaf area duration/senescence and crop productivity. In addition, targets for transgenic approaches used to manipulate leaf senescence are summarized, with a special focus on autoregulated expression of IPT as a mean to delay leaf senescence. As mentioned above, senescence can be induced by various abiotic stresses that have an enormous negative impact on global food production. Delaying leaf senescence should lead to crops that are tolerant or resistant to abiotic stresses. Gepstein and Glick (2013) summarize strategies that have been developed in recent years to improve crop protection against abiotic stresses, in particular drought and osmotic stresses. Many of these approaches target the metabolism of phytohormones, such as cytokinins, abscisic acid and ethylene, while others aim at reducing formation of reactive oxygen species or improving osmotic stress protection.
It is our hope that this special issue will keep the research community abreast with the most recent research advances in plant senescence. It may also serve as a textbook for advanced undergraduate students and graduate students.

Acknowledgments We would like to thank all authors for their excellent review articles for this special issue. Special thanks also goes to Dr. Wilhelm Gruissem, the Editor-in-Chief of Plant Molecular Biology, for his enthusiasm, encouragement and patience.

\section{References}

Gapper N, McQuinn R, Giovannoni J (2013) Molecular and genetic regulation of fruit ripening. Plant Mol Biol, this issue. doi:10. 1007/s11103-013-0050-3

Gepstein S, Glick B (2013) Strategies to ameliorate abiotic stressinduced plant senescence. Plant Mol Biol, this issue. doi:10.1007/ s11103-013-0038-z

Gregersen P, Culetic A, Boschian L, Krupinska K (2013) Plant senescence and crop productivity. Plant Mol Biol, this issue. doi: 10.1007/s11103-013-0013-8

Guo Y (2012) Towards systems biological understanding of leaf senescence. Plant Mol Biol, this issue. doi:10.1007/s11103-0129974-2

Hörtensteiner S (2012) Update on the biochemistry of chlorophyll breakdown. Plant Mol Biol, this issue. doi:10.1007/s11103-0129940-z

Humbeck K (2012) Epigenetic and small RNA regulation of senescence. Plant Mol Biol, this issue. doi:10.1007/s11103-012-0005-0

Jibran R, Hunter D, Dijkwel P (2013) Hormonal regulation of leaf senescence through integration of developmental and stress signals. Plant Mol Biol, this issue. doi:10.1007/s11103-0130043-2

Rogers HJ (2012) From models to ornamentals: how is flower senescence regulated? Plant Mol Biol, this issue. doi:10.1007/s11103-0129968-0

Tian S, Qin G, Li B (2013) Reactive oxygen species involved in regulating fruit senescence and fungal pathogenicity. Plant Mol Biol, this issue. doi:10.1007/s11103-013-0035-20

Zhang H, Zhou C (2012) Signal transduction in leaf senescence. Plant Mol Biol, this issue. doi:10.1007/s11103-012-9980-4 